\title{
Current Review; Biomarkers in Diagnosing Periprosthetic Joint Infection
}

\author{
George N. Guild III MD, *Alisina Shahi MD and Thomas L. Bradbury MD \\ Orthopaedic department at Emory University School of Medicine, USA
}

Received: October 14,2017; Published: October 20, 2017

*Corresponding author: Alisina Shahi MD, Clinical Research Department, Rothman Institute at Thomas Jefferson University, Sheridan Building, Suite 1000, 125 South $9^{\text {th }}$ Street, Philadelphia, PA 19107, USA, Tel: (484) 904-5799; Email: ali.sinair@gmail.com

\section{Mini Review}

Periprosthetic joint infection (PJI) is one of the most dreaded complications after total joint arthroplasty [1]. There is no gold standard for diagnosing PJI, hence, a clinician who encounters a suspected PJI case, ought to use a combination of tests. All of which, besides their expense can be invasive and even this can't provide $100 \%$ accuracy [2]. Several biomarkers have been introduced that are potentially reliable tools for diagnosing PJI [3]. In this article, we aimed to review the current diagnostic measures of PJI with a special focus on molecular biomarkers.

\section{Synovial Fluid Biomarkers}

Synovial fluid biomarkers play an imperative role in the diagnosing PJI. Leukocyte esterase (LE), human $\alpha$-defensin, human $\beta$-defensin, synovial CRP, and cathelicidin LL-37 are namely the biomarkers that have shown promising results. LE is an enzyme that is secreted by the activated neutrophils. It can be detected using colorimetric strip tests via reactions that result in a color change [4]. LE is a readily available and simple test and is now part of the minor criteria of the Musculoskeletal Infection Societydiagnostic criteria for PJI [5]. Tischler et al. [6] demonstrated that the LE strip test has a high specificity, positive, and negative predictive value for diagnosing PJI. Wetters et al. [7] investigated the accuracy of the LE test and reported a sensitivity of $92.9 \%$ to $100 \%$ and a specificity of $77.0 \%$ to $88.8 \%$. The important point is to note that bloody samples cannot be evaluated for the LE test without being centrifuged as the presence of blood can potentially interfere with the colorimetric changes of the test strip [6].

Synovial fluid $\alpha$-defensin test has shown great sensitivityand specificity for diagnosing PJI, 97\% and 96\% consequetively [8]. Defensins are 2-6 $\mathrm{kDa}$ cationic microbicidal peptides that are active against many Gram-negative and Gram-positive bacteria, fungi, and enveloped viruses [9]. Defensins in mammalians are classified into alpha, beta, and theta categories, based on their size and pattern of disulfide bonding. Alpha-defensins are particularly found in neutrophils, certain macrophage populations, and Paneth cells. Defensins are produced in response to microbial products or pro-inflammatory cytokines. The $\alpha$-defensin mechanism by which microorganisms are killed and inactivated is not yet fully understood. Nevertheless, it is thought that it causes membrane disruption in microorganisms [10]. The spatially separated, charged, and hydrophobic regions, along with the polar topology of $\alpha$-defensin, allows it to insert itself into the membranes; therefore, the hydrophobic regions are buried within the interior phospholipid membrane and the cationic sites interact with anionic phospholipid head groups and water. The disruption of membrane integrity and function leads to lysis of the microorganisms [11,12]. Several studies have endorsed the role of the $\alpha$-defens in test in diagnosing PJI. The $\alpha$-defensin test provides consistent results regardless of the organism type, Gram staining, species, or virulence of the organism [13].

CRP, which elevates in both the serum and synovial fluid of PJI cases, is a protein that is synthesized by the liver in response to acute inflammation [14]. Parvizi et al. [15] reported a statistically significant difference in the mean of synovial fluid CRP comparing septic and aseptic patients; $40 \mathrm{mg} / \mathrm{L}$ vs. $2 \mathrm{mg} / \mathrm{L}$, respectively $(\mathrm{p}<.0001)$. The study found a sensitivity of $85 \%$ and a specificity of $95 \%$ when $9.5 \mathrm{mg} / \mathrm{L}$ was considered as the threshold.

Human host defense peptide LL-37 is an antimicrobial peptide that induces mediators such as IL-8 and regulates the inflammatory response [16,17]. Gollwitzer et al. [18] reported that LL-37 has a sensitivity of $80 \%$ and specificity of $85 \%$, with an area under the curve of 0.875 for diagnosing PJI.

\section{Serum Biomarkers}

Serum markers are favorable diagnostic tools due to their accessibility and low-risk nature. The American Academy of Orthopaedic Surgeons and the International Consensus meeting on PJI currently recommend using serum erythrocyte sedimentation rate (ESR) and C-reactive protein (CRP) as the first line diagnostic work up for patients with suspected PJI. Reports have shown a 
sensitivity of $91 \%$ and specificity of $72 \%$ for ESR and a sensitivity of $94 \%$ and specificity of $74 \%$ for CRP [19-21]. Serum ESR and CRP are well-known biomarkers that indicate systemic inflammatory response [22]. However, these markers are elevated with any type of inflammation and/or infection, compromising their specificity for diagnosis of PJI. Recent evidence suggests that PJI with slowgrowing organisms may not result in a fully-fledged physiological response and hence may not result in elevation of ESR and CRP in the serum, raising a concern for the sensitivity of these tests. Furthermore, a study by our group showed that the administration of systemic antibiotics can significantly compromise the results of these laboratory values [2]. It is imperative for clinicians to consider the timing of infection prior to assessing patients' ESR and CRP results, as these markers are frequently elevated in the early postoperative period. Studies have shown that ESR can be elevated up to 6 weeks after surgery and CRP can be elevated for up to 2 weeks [23].

Procalcitonin (PCT) is a serum biomarker that elevates in the presence of bacteria. Bottner et al. [24] measured serum levels of several biomarkers including: PCT, interleukin (IL)-6, tumor necrosis factor (TNF)- $\alpha$, ESR, and CRP in 78 patients undergoing revision arthroplasty for PJI. The sensitivity of CRP and IL-6 were the highest (95\%) when the levels were greater than $3.2 \mathrm{mg} /$ $\mathrm{dL}$ and $12 \mathrm{pg} / \mathrm{mL}$, respectively. The authors recommended that combination of CRP and IL- 6 could be used as a screening test for PJI. PCT levels $(>0.3 \mathrm{ng} / \mathrm{mL})$ were very specific $(98 \%)$ but had a low sensitivity (33\%) for diagnosing PJI. In another study by Hügle et al. [25] authors showed that PCT with a threshold of $0.25 \mathrm{ng} /$ $\mathrm{mL}$ has a higher sensitivity and specificity for diagnosing septic arthritis than CRP, with a sensitivity of $93 \%$ and specificity of $75 \%$. This could possibly be rationalized by the fact that PCT is secreted by the mononuclear phagocyte system only when stimulated by lipopolysaccharide. Therefore, PCT can be a useful tool to differentiate between bacterial infections of the joint and other causes of inflammation. Nevertheless, more recent studies claimed that PCT is not a very accurate tool for diagnosing PJI [26,27].

IL-6 is another serum marker that has gained attention for diagnosing PJI. IL-6 is secreted by different immune cells and triggers the excretion of CRP; therefore, it is believed that the IL-6 levels rise much faster than CRP and has been reported to be a sensitive marker for diagnosing PJI, however it also lacks specificity [24,28]. (p-6) Wirtz et al. [29] advocated the role of IL-6, and in their study authors showed that IL- 6 is a better indicator for postoperative inflammatory response than CRP in patients undergoing TJA.

There is a dire need for a sensitive and specific serum biomarker for diagnosing PJI and numerous efforts have been made to pursue this goal. Serum D-dimer is another biomarker that has shown very promising results for diagnosing PJI. Shahi et al. [30] in their prospective study showed that serum D-dimer outperforms both ESR and CRP for diagnosing PJI with a sensitivity of $89 \%$ and a specificity of $93 \%$.
Modern medicine has entered a new era where molecular biomarkers play an increasingly important role for diagnosis of various conditions. PJI is no exception and new biomarkers hold great promise for it. Efforts should continue to hopefully find a gold standard test for a timely diagnosis of this serious complication.

\section{References}

1. Shahi A, Parvizi J (2015) Prevention of periprosthetic joint infection: pre, intra, and postoperative strategies. Med Flum 51(1): 103-113.

2. Shahi A, Deirmengian C, Higuera C, Chen A, Restrepo C, et al. (2015) Premature Therapeutic Antimicrobial Treatments Can Compromise the Diagnosis of Late Periprosthetic Joint Infection. Clin Orthop 437(7): 2244-2249.

3. Deirmengian C, Kardos K, Kilmartin P, Cameron A, Schiller K, et al. (2014) Diagnosing periprosthetic joint infection: has the era of the biomarker arrived? Clin Orthop 472(11): 3254-3262.

4. Parvizi J, Jacovides C, Antoci V, Ghanem E (2011) Diagnosis of periprosthetic joint infection: the utility of a simple yet unappreciated enzyme. J Bone Joint Surg Am 93(24): 2242-2248.

5. Parvizi J, Gehrke T (2014) International Consensus Group on Periprosthetic Joint Infection. Definition of periprosthetic joint infection. J Arthroplasty 29(7): 1331.

6. Tischler EH, Cavanaugh PK, Parvizi J (2014) Leukocyte Esterase Strip Test: Matched for Musculoskeletal Infection Society Criteria. J Bone Jt Surg Am 96(22): 1917-1920.

7. Wetters NG, Berend KR, Lombardi AV, Morris MJ, Tucker TL, et al. (2012) Leukocyte esterase reagent strips for the rapid diagnosis of periprosthetic joint infection. J Arthroplasty 27(8): 8-11.

8. Deirmengian C, Kardos K, Kilmartin P, Cameron A, Schiller K, et al. (2014) Combined measurement of synovial fluid $\alpha$-Defensin and C-reactive protein levels: highly accurate for diagnosing periprosthetic joint infection. J Bone Joint Surg Am 96(17): 1439-1445.

9. White SH, Wimley WC, Selsted ME (1995) Structure, function, and membrane integration of defensins. Curr Opin Struct Biol 5(4): 521-527.

10. Mathew B, Nagaraj R (2015) Antimicrobial activity of human $\alpha$-defensin 5 and its linear analogs: $\mathrm{N}$-terminal fatty acylation results in enhanced antimicrobial activity of the linear analogs. Peptides 71: 128-140.

11. Xie Z, Feng J, Yang W (2015) Human $\alpha$-defensins are immune-related Kv1.3 channel inhibitors: new support for their roles in adaptive immunity. faseb J Off Publ Fed Am Soc Exp Biol 29(10): 4324-4633.

12. Wiesner J, Vilcinskas A (2010) Antimicrobial peptides: the ancient arm of the human immune system. Virulence 1(5): 440-464.

13. Bingham J, Clarke H, Spangehl M, Schwartz A, Beauchamp C (2014) The alpha defensin- 1 biomarker assay can be used to evaluate the potentially infected total joint arthroplasty. Clin Orthop 472(12): 4006-4009.

14. Parvizi J, Jacovides C, Adeli B, Jung KA, Hozack WJ (2012) Mark B Coventry Award: synovial C-reactive protein: a prospective evaluation of a molecular marker for periprosthetic knee joint infection. Clin Orthop 470(1): 54-60.

15. Parvizi J, McKenzie JC, Cashman JP (2012) Diagnosis of periprosthetic joint infection using synovial C-reactive protein. J Arthroplasty 2012: 27(8): 12-16.

16. Nijnik A, Hancock REW (2009) The roles of cathelicidin LL-37 in immune defences and novel clinical applications. Curr Opin Hematol 16(1): 4147.

17. Overhage J, Campisano A, Bains M, Torfs ECW, Rehm BHA, et al. (2008) Human host defense peptide LL-37 prevents bacterial biofilm formation. Infect Immun 76(9): 4176-4182. 
18. Gollwitzer H, Dombrowski Y, Prodinger PM, Peric M, Summer B, et al. (2013) Antimicrobial peptides and proinflammatory cytokines in periprosthetic joint infection. J Bone Joint Surg Am 95(7): 644-651.

19. Gehrke T, Parvizi J (2014) Proceedings of the International Consensus Meeting on Periprosthetic Joint Infection. J Arthroplasty 29(2): 4.

20. Parvizi J, Adeli B, Zmistowski B, Restrepo C, Greenwald AS (2012) Management of periprosthetic joint infection: the current knowledge: AAOS exhibit selection. J Bone Joint Surg Am 94(14): 104.

21. Austin MS, Ghanem E, Joshi A, Lindsay A, Parvizi J (2008) A simple, cost-effective screening protocol to rule out periprosthetic infection. J Arthroplasty 23(1): 65-68.

22. Gabay C, Kushner I (1999) Acute-Phase Proteins and Other Systemic Responses to Inflammation. N Engl J Med 340(6): 448-454.

23. Parvizi J, Valle CJD (2010) AAOS Clinical Practice Guideline: Diagnosis and Treatment of Periprosthetic Joint Infections of the Hip and Knee. J Am Acad Orthop Surg 18(12): 771-772.

24. Bottner F, Wegner A, Winkelmann W, Becker K, Erren M, et al. (2007) Interleukin-6, procalcitonin and TNF- $\alpha$. Bone Jt J 89(1): 94-99.

25. Hügle T, Schuetz P, Mueller B, Laifer G, Tyndall A, et al. (2008) Serum procalcitonin for discrimination between septic and non-septic arthritis. Clin Exp Rheumatol 26(3): 453-456.
26. Worthington T, Dunlop D, Casey A, Lambert R, Luscombe J, et al. (2010) Serum procalcitonin, interleukin-6, soluble intercellular adhesin molecule-1 and IgG to short-chain exocellular lipoteichoic acid as predictors of infection in total joint prosthesis revision. Br J Biomed Sci 67(2): 71-76.

27. Drago L, Vassena C, Dozio E, MM Corsi, E De Vecchi et al. (2011) Procalcitonin, C-reactive protein, interleukin-6, and soluble intercellular adhesion molecule-1 as markers of postoperative orthopaedic joint prosthesis infections. Int J Immunopathol Pharmacol 24(2): 433-440.

28. Selberg O, Hecker H, Martin M, Klos A, Bautsch W, Köhl J (2000) Discrimination of sepsis and systemic inflammatory response syndrome by determination of circulating plasma concentrations of procalcitonin, protein complement 3a, and interleukin-6. Crit Care Med 28(8): 27932798.

29. Wirtz DC, Heller KD, Miltner O, Zilkens KW, WolffJM (2000) Interleukin-6: a potential inflammatory marker after total joint replacement. Int Orthop 24(4): 194-196.

30. Shahi A, Kheir MM, Tarabichi M, Hosseinzadeh HRS, Tan TL, et al. 2017 Serum D-Dimer Test Is Promising for the Diagnosis of Periprosthetic Joint Infection and Timing of Reimplantation. J Bone Joint Surg Am 99(17): 1419-1427.

Assets of Publishing with us
- Global archiving of articles
RESEARCHES

